\title{
La Ciudad y sus Interacciones con la Dimensión Energética y Ambiental
}

\author{
Dr. Carlos Discoli \\ Dra. Irene Martini \\ Instituição : IIPAC- FAU- UNLP
}

\begin{abstract}
Resumen
El trabajo evalúa el estado de situación de las aglomeraciones urbanas intermedias de la Argentina relacionando aspectos del hábitat urbano, su infraestructura energética y el ambiente. Propone un análisis integrado, abierto y flexible que incluye la diversidad y calidad de los servicios urbanos y el estado ambiental de la ciudad. Considera información cuali-cuantitativa global y desagregada; y su análisis se orienta a brindar información calificada para dar respuestas a parte de los crecientes problemas de diagnóstico y gestión de las ciudades intermedias de nuestra región. Entre las aplicaciones permite identificar y evaluar áreas vulnerables como consecuencia de los desequilibrios generados por el crecimiento urbano espontáneo. Para este trabajo se aborda el sector residencial, y se evalúa la ocupación del territorio, su crecimiento, sus necesidades energéticas y sus consecuencias ambientales. Los resultados muestran mapas conformando zonas homogéneas que verifican el grado de equidad de las variables consideradas.
\end{abstract}

\begin{abstract}
1. Introducción
Los efectos de la globalización y los problemas energéticos-ambientales desencadenados a partir de los años 070 han impactado significativamente en los procesos de organización y reorganización del territorio, así como en la degradación del hábitat y la presión sobre los recursos naturales. Entre las consecuencias principales podemos mencionar la fragmentación socio-espacial, en particular en el ámbito urbano y suburbano, estableciéndose significativos desequilibrios e inequidades fundamentalmente en los paises menos desarrollados.
\end{abstract}

En la Argentina las aglomeraciones urbanas no escapan a este proceso de fragmentación agravada por administraciones poco eficaces; por información dispersa y fragmentada; y por la falta de instrumentos apropiados para sistematizar datos y elaborar diagnósticos acertados. Las consecuencias implican problemas de desequilibrio socio-económicos y socio-energéticos, llegando a situaciones de sobre-explotación indiscriminada de los recursos, de baja habitabilidad de inequidad en los gastos, y de una degradación insostenible del ambiente.

Para este trabajo consideramos como campo de aplicación al área metropolitana del Gran La Plata, capital de la Provincia de Buenos Aires. Esta ciudad fue concebida en 1882 como la primera ciudad contemporánea planificada de la Argentina. Su crecimiento posterior y principalmente fuera del casco urbano fundacional, se rigió en general por intervenciones no planificadas. Se modificaron sustantivamente los lineamientos originales de la ciudad y el crecimiento aconte- 
cido hasta el momento. Ese crecimiento periurbano alteró los preceptos higienistas originales que habían ordenado el proyecto de la ciudad, basándose en su trazado, estructura y calidad de los sistemas urbanos principales. Esas transformaciones socio-espaciales alteraron su génesis compacta, modificándose en el tiempo en sistemas degradados de borde y núcleos satélites de baja densidad.

A escala urbana, en muchos casos, el deterioro se manifiesta en un desarrollo casi incontrolado y en cierto aspecto caótico, tanto en los aspectos físicos-ambientales como socio-económicos. Estos procesos se desarrollaron con patrones que no tuvieron en cuenta, entre otras cosas, las oportunidades emergentes del clima y del ambiente. Estas zonas urbanas se caracterizaron por su escasa y precaria existencia de servicios y su consecuente baja calidad y eficiencia. Todo ello "producto por un lado de la carencia de recursos e insuficiente inversión en infraestructura y por otro de los condicionamientos de los gobiernos locales en su capacidad de planificar, coordinar y administrar la operación de crecimiento de las ciudades". ("Programa conjunto UNDP/ Banco Mundial/UNCHS, Hábitat, 1991).

Si bien en la actualidad existen esfuerzos gubernamentales para revertir dichas situaciones, las transformaciones han sido tan profundas y vertiginosas, que se hacen notar aún las dificultades que tienen los decisores de gestión para detectar y cuantificar eficazmente las distorsiones urbanas.

En este contexto se ha desarrollado e implementado una metodología en la que intervienen estrategias convergentes de análisis apuntando a un fin común (C. Discoli 2003), (C. Discoli et al. 2006a). El desarrollo e implementación de diferentes técnicas y modelos tendientes a relacionar la diversidad de variables, permitió evaluar la dinámica urbana, su crecimiento y la interacción con el ambiente y la calidad de vida urbana.

La metodología utilizada propone transformar la visión tradicional de gestión urbana con el objeto de instrumentar politicas que incluyan acciones inovativas tendientes a generar cambios estructurales en el marco global de lo que se denomina ciudades ambientalmente sanas. Dichas metas se encuentran en consonancia con las elaboradas inicialmente en la Conferencia Mundial sobre Medio Ambiente y Desarrollo, Rio 092 , donde fue aprobada la Agenda XXl, en el marco del desarrollo sustentable a escala mundial. En ella se planteó la necesidad de elaborar metodologías apropiadas para la realización acertada de diagnósticos, evaluación y adopción de decisiones, replantear escenarios alternativos y reformular políticas energéticas. (Conferencia Mundial So- bre el Medio Ambiente, Agenda XXI. 1992).

En este contexto, este trabajo plantea analizar las principales variables urbanas, en particular las socio-energéticas, sus consecuencias socio-espaciales y la opinión de los usuarios sobre los fenómenos involucrados a partir de un modelo cuali-cuantitativo de calidad de vida urbana (Discoli, C. et al., 2006b) y (Discoli, C., et al., 2007). Para tal fin se conforman mapas de calidad estableciendo zonas homogéneas que identifican los aspectos de la oferta de los servicios urbanos a través de la infraestructura y de la cobertura, asi como los requerimientos de la demanda considerando la percepción de los usuarios. Como resultado del trabajo se presentan las áreas con mayor vulnerabilidad urbana, consecuencia del crecimiento espontáneo en concordancia con las mayores carencias en cuanto al control urbano. Estos permitirian confirmar la trayectoria de insustentabilidad creciente que hemos detectado en la aglomeración en estudio, en nuestro caso el Gran La Plata.

\section{Evaluación e Identificación de Áre- as de Mayor Vulnerabilidad Urbana}

Para el desarrollo del trabajo se plantea evaluar las áreas de mayor vulnerabilidad urbana a partir de los desequilibrios generados por el crecimiento residencial espontáneo, y sus consecuencias sobre la sustentabilidad de los servicios urbanos de indole energética. Para ello se plantea abordar los siguientes aspectos:

- El Sector Residencial: Se evalúa la ocupación en el territorio, y su evolución en cuanto al crecimiento, sus necesidades energéticas y sus respectivas emisiones de contaminantes

- Los Servicios Básicos de Infraestructura: Consideramos en este caso los relacionados a cubrir la demanda energética urbana (Energía Eléctrica y Gas Natural). Se establecen perfiles expresados en mapas con niveles de calidad de cada servicio, donde se relaciona la oferta a través de cualidades que la caracterizan, con las coberturas de cada red y la opinión de los usuarios (la demanda).

A continuación se analiza un cuerpo de variables básicas, a los efectos de exponer la mecánica de procedimientos utilizada en la metodología; y la visualización de sus interacciones a través de mapas urbanos, muestra la potencialidad de la misma en cuanto a la diversidad de respuestas posible.

\subsection{El Sector Residencial}

La población en general se encuentra asimétricamente distribuida en el territorio, y su grado de 
ocupación y niveles de asentamientos conforman lo que denominamos el sector Residencial. Se plantea analizar su distribución en el territorio. remarcando la ocupación y su evolución en el tiempo. Esto nos permite identificar y analizar con mayor desagregación los perfiles de demanda en consonancia con la dinámica de crecimien:o real, mejorando así la calidad de los servicios en cuanto a los aspectos energéticos, sociales y económicos.

E| crecimiento de la población se muestra en la figura 1 ; y en las figuras 2 y 3 se muestra por ejemplo la evolución en el territorio de dos décadas con significativa fragmentación socio-econó mica. En las mismas se verifica un incremento de población con mayores niveles de ocupación en aquellas zonas periurbanas y sub-urbanas de baja consolidación. La aceleración de dicha ocupación se debió principalmente a ciertos atractores como el menor costo de la tierra, la presencia mínima y/o cercana de alguna red de infraestructura básica (Energía Eléctrica), y cierto grado de accesibilidad (trazado de calles de tierra o mejoradas y cierta cercanía a los medios de transporte público). En cambio, en las áreas ya urbanizadas se registró una variación general relacionada básicamente con el crecimiento vegetativo.

Conocer la ocupación del territorio y su densidad poblacional nos permite evaluar las necesidades

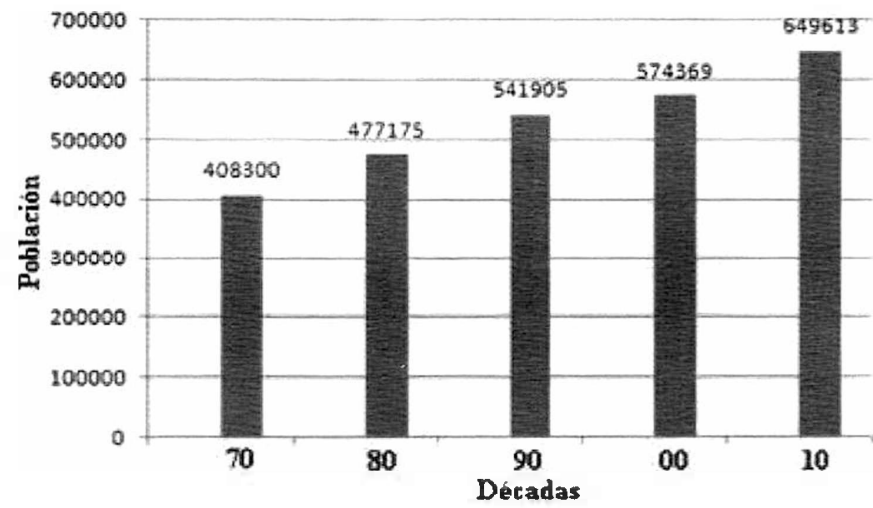

Figura 1: Perfil de crecimiento de la Población.

=iente: INDEC y Municipalidad de La Plata.

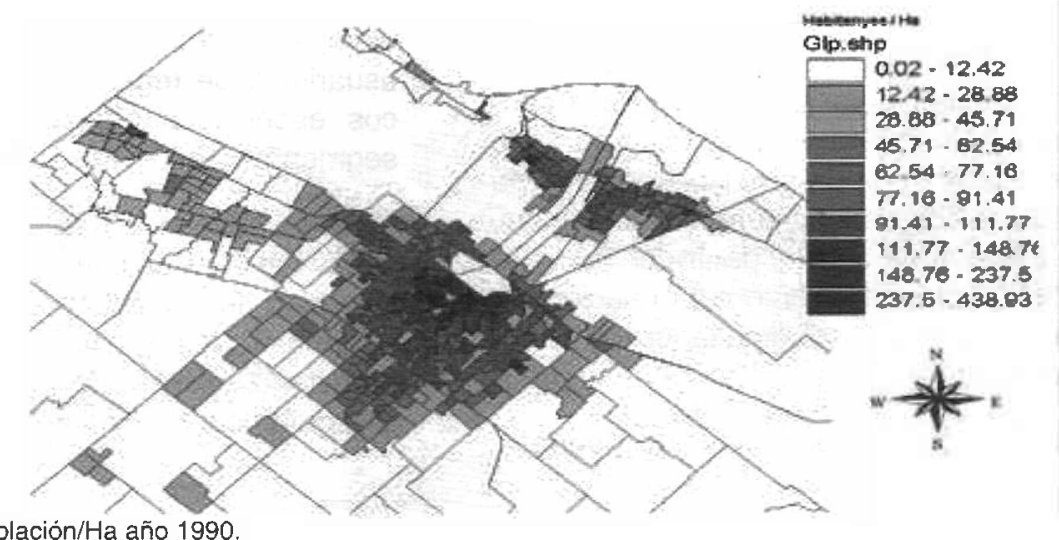

Figura 2: Densidad de población/Ha año 1990.

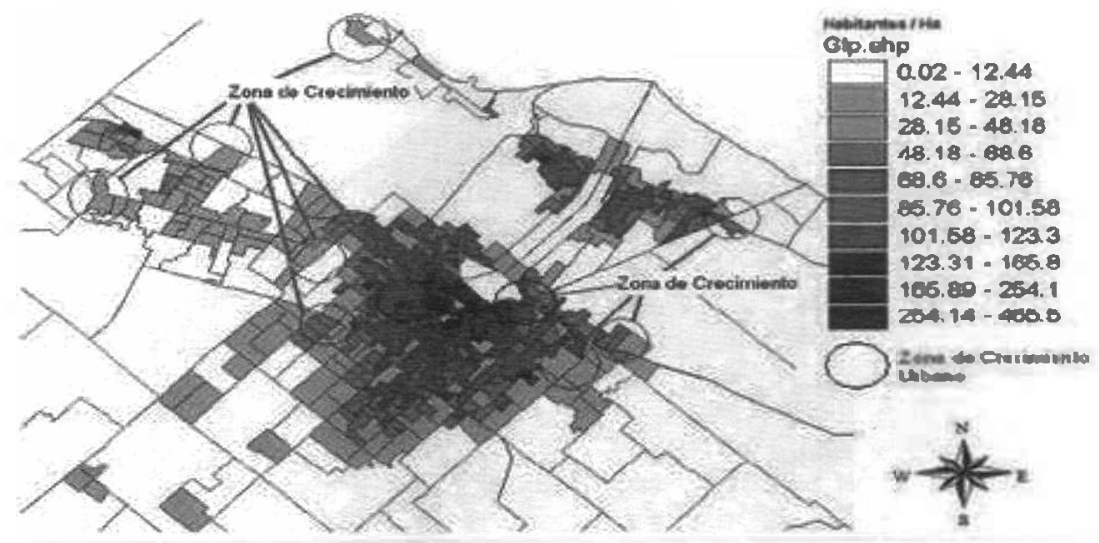

Fgura 3: Densidad de población/Ha año 2000. 
energéticas y su consecuente contaminación.

\section{a- Demanda energética del Sector Residencial}

Para caracterizar la demanda energética del sector residencial se utilizaron encuestas estructuradas de hogares en las diferentes décadas que permitieron verificar y contrastar la estructura social del área y establecer algunos patrones de consumo (AUDIBAIRES, 1986), (URE-AM, 2000), (Rosenfeld, Y. et al., 2000). Estos patrones están asociados a lo que se denomina la gestión del consumo, donde algunos estudios han verificado diferentes modos de uso del equipamiento entre diferentes hogares y entre padres e hijos, e inclusive entre géneros. (Desjeux, D. et al. 1996).

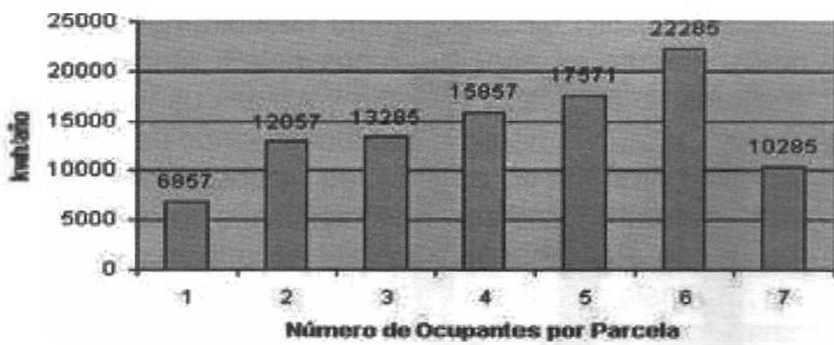

Figura 4: Consumo por parcela y por ocupante.

Para tal fin, se relacionaron los aspectos sociales a través de la estructura familiar, el número de ocupantes y su situación laboral con los consumos energéticos totales y su distribución en el territorio.

El consumo promedio por hogar (parcela) y cantidad de ocupantes se muestra en la figura 4 . Se verifica un aumento progresivo del consumo de energía con relación al número de ocupantes, donde se observa un corte a partir del $7 \mathrm{mo}$. habitante. Dicho comportamiento está relacionado principalmente con el uso simultáneo de espacios y artefactos.

La caracterización de consumos fue georrefe- renciada en el territorio en forma detallada en un sistema de información geográfica (Arq Gis 9) en donde se identificaron los hogares encuestados. Esto permitió formular, en función de las unidades territoriales urbanas (Radio censal, Fracción, Manzana), diferentes situaciones de demandas energéticas. A partir de las demandas reales se pueden formular perfiles de consumo territoriales; y establecer relaciones en cuanto a la disponibilidad/indisponibilidad de los servicios en cada zona urbana. La información analizada hasta el momento nos permitió establecer los primeros perfiles de consumo de energía por hogar y la consecuente contaminación generada. Como

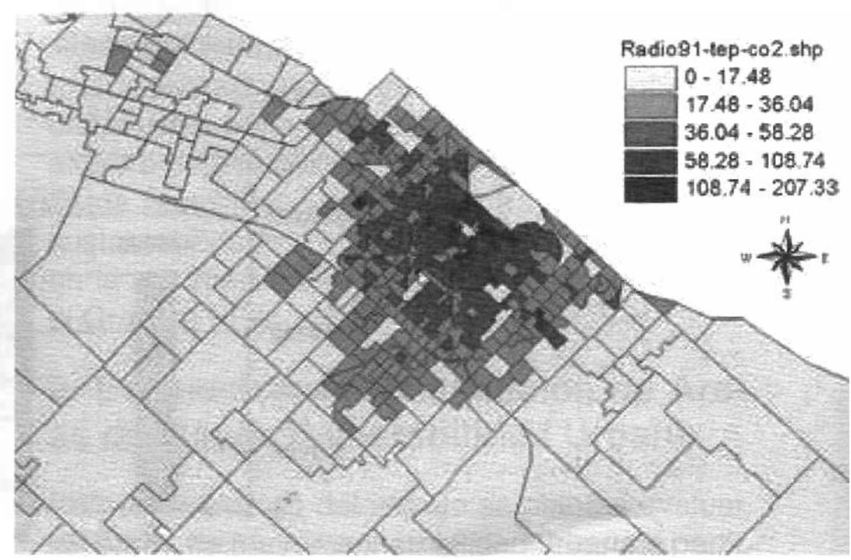

Figura 6: Densidad Energética total de Sector Residencial por radio censal. TEP/Ha.año.

ejemplo en la figura 5 se localiza la muestra de usuarios y se registran los consumos energéticos específicos anuales, clasificados en tres segmentos (Bajos, Medios y Altos).

Si completamos y relacionamos la información relativa al perfil de consumo por habitante, con la densidad de población residente y localizada en el territorio, los resultados de dicha interacción, permiten evaluar la densidad energética total de la región desde el lado de la demanda por habi-

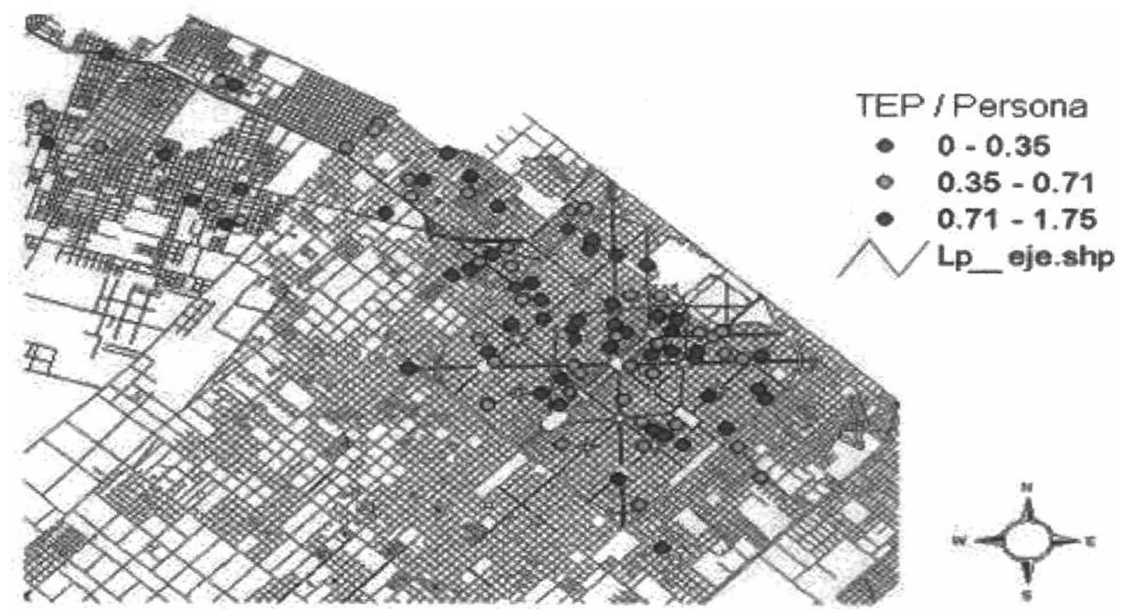

Figura 5: Consumo total. Localización de hogares encuestados. Fuente: G1. IIPAC. 
:ante y por año. La figura 6 muestra el mapa de a región con la densidad energética del sector residencial en TEP/habitante año.

A partir de este análisis se establecen zonas con niveles de demanda concordantes con las diferentes consolidaciones urbanas. También se dentifican zonas dispersas con niveles de consumo diferenciados coincidentes con las áreas céntricas de algunas delegaciones municipales periurbanas (núcleos satélites), y localizaciones puntuales de muy alta densidad.

La obtención de índices específicos localizados en el territorio nos permite evaluar las áreas urbanas con mayor precisión. En el caso de los aspectos energéticos, podemos conocer la situación actual y/o dimensionar la potencial deman- da del sector residencial a partir de la dinámica de asentamientos urbanos. El crecimiento está fuertemente influenciado por los atractores mencionados (costo de la tierra, acceso cercano a laguna red de energia y accesibilidad). En consecuencia, inferir en la dinámica de crecimiento a partir de ellos nos permitiría estimar la posible demanda energética potencial.

\section{b- Consecuencias ambientales por parte de la demanda energética.}

Si analizamos las implicancias ambientales relacionadas al consumo de energía en el sector residencial, vemos que la metodología de trabajo propuesta nos permite cuantificar la contaminación por emisiones aéreas producto de la demanda localizada. Para ello se calculan y geor-

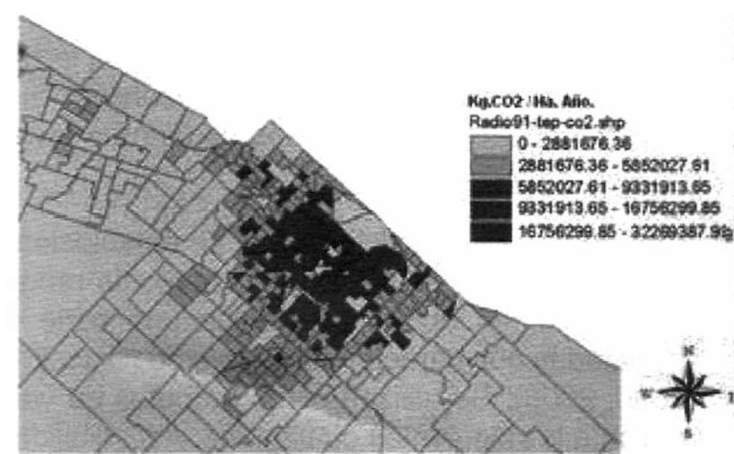

Figura 7: Emisiones de $\mathrm{CO} 2$ Totales. Sector Residencial. $\mathrm{Kg}$ CO2/Ha.año.

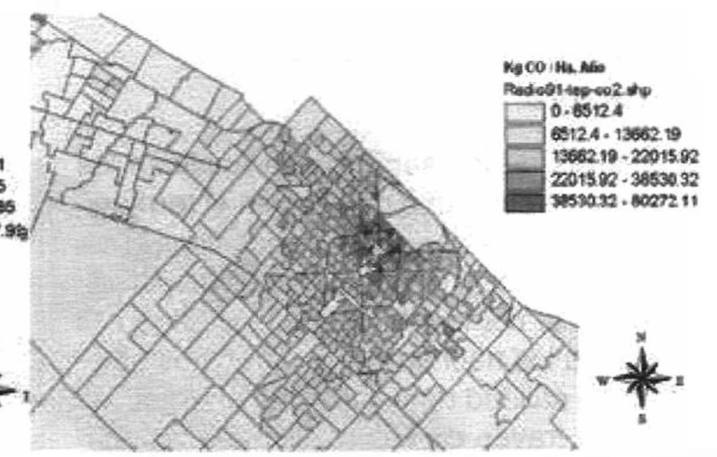

Figura 8: Emisiones de CO Total. Sector Residencial. Kg CO/ Ha.año.

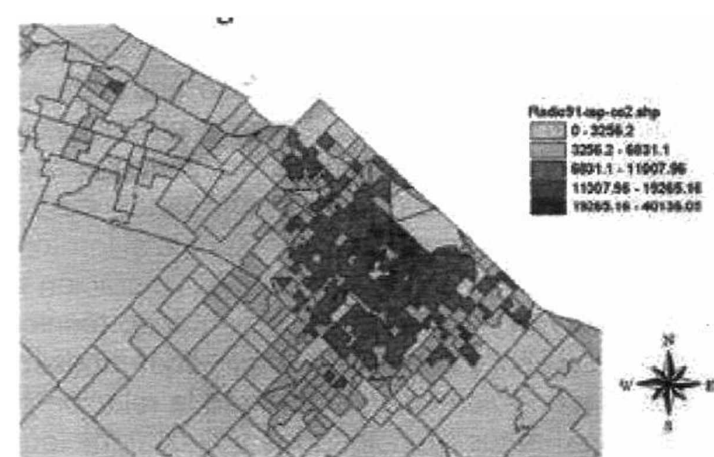

Figura 9: Emisiones de NOx Totales. Sector Residencial. Kg NOx/Ha.año.

referencian los contaminantes aéreos primarios (CO2, CO, NOx y SOx), emitidos por la combustión de los vectores energéticos predominantes en el sector Residencial (Gas natural por red y gas envasado). No se consideraron en este caso las emisiones indirectas provocadas por la generación de energía eléctrica dado que no están generadas en la región de análisis. Las figuras $7,8,9$ y 10 muestran el nivel de emisiones de algunos de los contaminantes y su localización en el territorio en $\mathrm{Kg}$ de contaminante por manzana (Ha) y por año. La correspondencia entre consumos y emisiones muestra una vez más, las zonas urbanas más comprometidas.

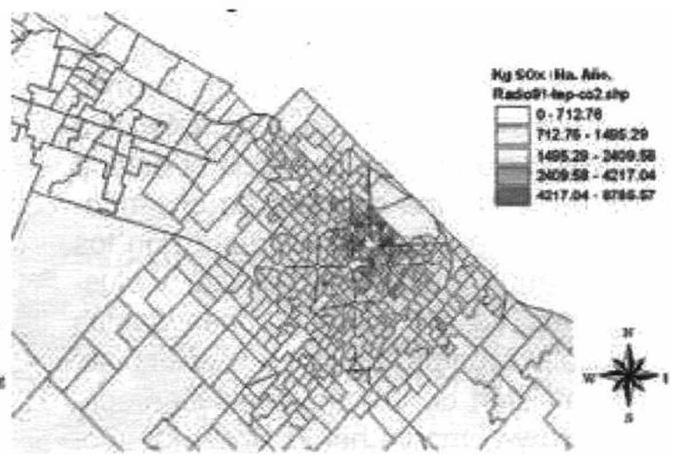

Figura 10: Emisiones de SOx Totales. Sector Residencial. $\mathrm{Kg} \mathrm{SOx/Ha.año.}$

Para concluir con los aspectos relacionados a la demanda energética del sector residencial, se sintetizaron en la tabla 1 los consumos energéticos y las emisiones especificas por habitante y por zonas urbanas.

La información obtenida hasta el momento nos permite dimensionar el estado del arte de la demanda y sus consecuencias ambientales directas. Estos resultados georreferenciados pueden ser contrastados con la oferta energética provista por las empresas de servicios a los efectos de evaluar los desequilibrios generados por el crecimiento residencial espontáneo, y sus con- 
Tabla 1: Consumos energéticos y emisiones especificas por habitante y por zona urbana.

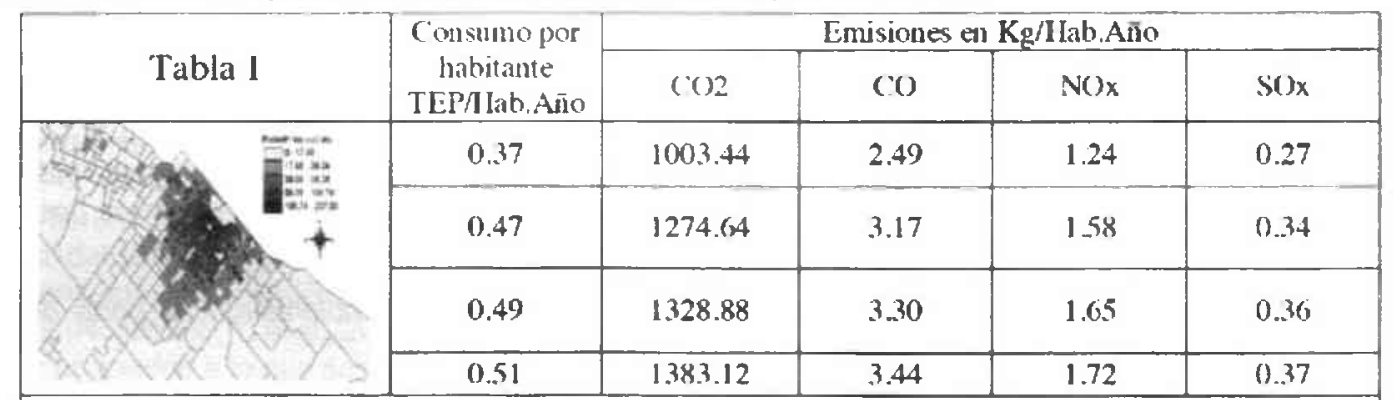

Las emisiones aéreas de los contaminantes primaros se calcularon a partir de los valores de conversión establecidos por el IPCC

secuencias sobre la sustentabilidad actual de los servicios de índole energética. Para tal fin se analiza en este trabajo el servicio eléctrico y el servicio de gas distribuido por red.

\subsection{Los Servicios Básicos de Infraestructura}

Para el análisis de los servicios se analizan las interacciones de cada uno de ellos en el territorio a través de sus características especificas (Discoli, C. 2003), (Discoli, C. et al., 2006) (Discoli, C. et al., 2008). Así como conocemos los aspectos energéticos de los habitantes, podemos establecer niveles de calidad de las fuentes energéticas disponibles, a través de sus cualidades, (atributos de valoración), sus áreas de influencia (coberturas en el territorio), su utilización y el grado de aceptación de los usuarios expresado por medio de la opinión (inconvenientes delectados). Su localización y distribución geográfica nos permite cotejar las áreas de cubrimiento, y establecer a partir de la valoración de los servicios el estado de la oferta de cada uno de ellos en el territorio. Los resultados pueden compararse con los mapas de demanda energética, verificar y ajustar desequilibrios, así como inferir zonas viables para implementar fuentes sustitutas más limpias, minimizar la emisión de contaminantes aéreos. De esta manera y como ya hemos mencionado, se pueden establecer vulnerabilidades a partir de los desajustes de cada red de servicio (entre ofertas y demandas no previstas) y fundamentalmente en aquellos sectores que, por defecto, utilizan fuentes energéticas sustitutas por carecer de acceso a las redes. Los resultados en términos de calidad ofertada por los servicios energéticos se integran con los energéticos-sociales, (demanda) utilizando como soporte al territorio. Esta integración permite identificar con claridad los desequilibrios entre los servicios y sus usuarios manteniendo el origen de sus causas. Los servicios energéticos de uso frecuente en el área de estudio son:

\section{a- Servicio de Energía Eléctrica (EE)}

Para evaluar el servicio de EE, se desarrollaron trabajos inherentes a definir la calidad, por medio de un análisis en el que interviene: la evaluación de sus cualidades, a partir de ponderaciones relativas, considerando las distintas fuentes energéticas utilizadas en el área de estudio; la territorialización de su cobertura o área de influencia y el grado de aceptación de los usuarios a través de su opinión.

Para calificar el servicio de EE se establecieron mecanismos de valoración por medio de rangos numéricos, en donde se califica una serie de cualidades que caracterizan al mismo (Discoli, C. et al., 2006). Se utilizan como atributos de valoración a las siguientes cualidades: Accesibilidad, Continuidad, Costo, Traslado-manipuleo, Riesgo de utilización, Existencia de organismos/empresas que respalden técnica y administrativamente el servicio y contaminación. Los servicios que implementen un mayor consenso entre los atributos mencionados, a criterio de personal calificado, son los que obtendrán mayor calificación y en consecuencia podrán definir tendencias de calidad como información transferible al territorio. Para perfeccionar y completar la valoración de cada servicio, se están implementando técnicas de lógica difusa con el objeto de poder modelizar con mayor precisión y objetividad la relación entre los atributos mencionados y las valoraciones obtenidas.

Para la determinación de las áreas de cobertura se utiliza información cartográfica digitalizada en SIG (ArcGis 9). Se calculan los indicadores de cubrimiento para el área en estudio y su representación territorial en forma porcentual, conformándose así tramas de cubrimiento. Los porcentajes obtenidos se normalizan estableciendo un valor ponderado, obteniendo así un factor de Área de Cobertura. (Figura 11). En este caso la cobertura representa el $100 \%$ del territorio habitado.

Para evaluar la aceptación del servicio de EE por los usuarios, se consideró en primera instancia la opinión general emitida por la encuesta de hoga- 
res en cuanto al servicio en su conjunto. Se procesó estadísticamente estableciendo un gráfico de aceptación segmentado en cinco categorías de opinión (Muy Bueno, Bueno, Regular, Malo y Muy Malo). Para obtener un factor de opinión se normalizan las 5 categorías asignando un valor numérico cuyo rango es de 0 a 1 . La especialización de los resultados por medio de la localización de cada valoración permite establecer los mapas de opinión. (Figura 12).

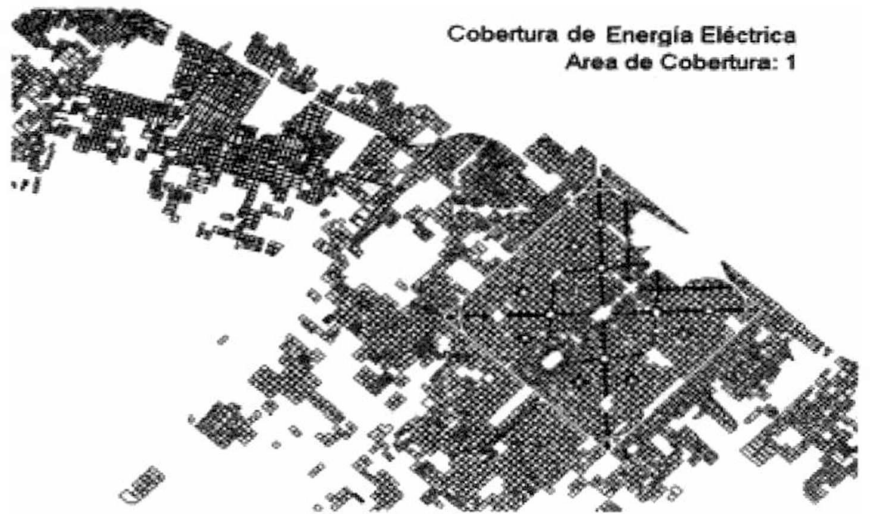

Figura 11: Cobertura de Energia Eléctrica. 100\% de cobertura.

=ente: G1. IIPAC.

Figura 12: Áreas homogéneas de opinión. Red de EE.

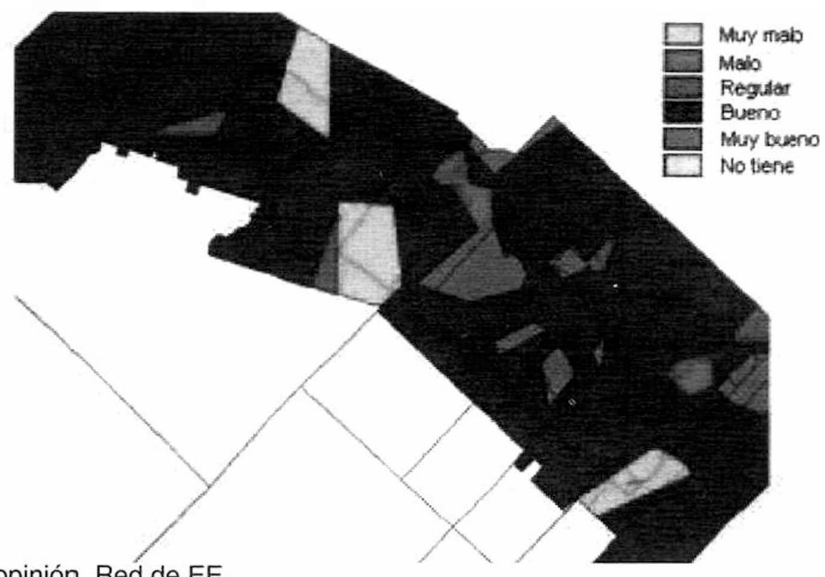

Fuente: G1. IIPAC.

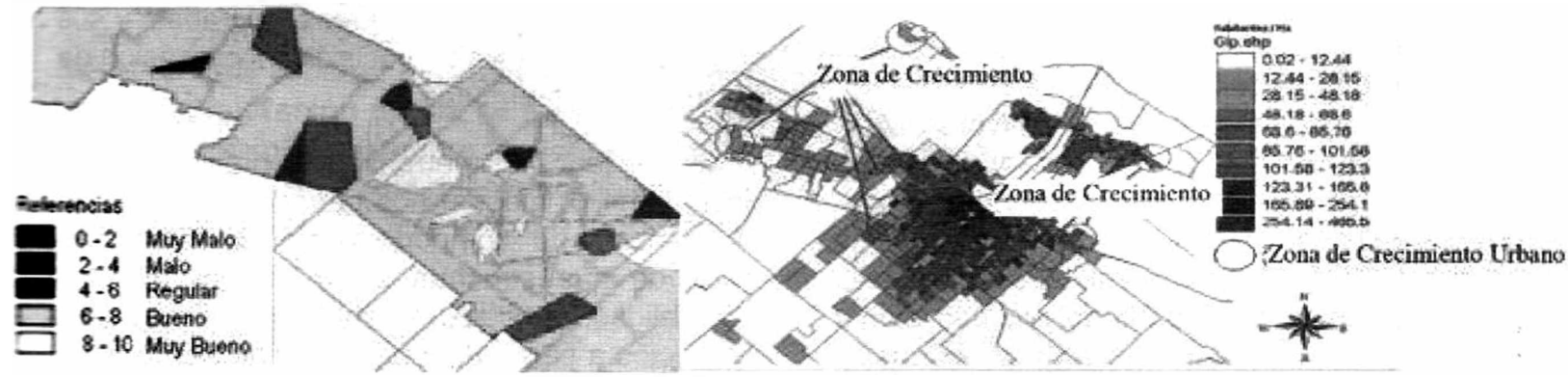

Figura 13: Perfil de Calidad del Servicio E.E. Inequidades coincidentes.

toaptado de: G1. IIPAC

Luego de valorar el servicio y establecer su cosertura y el grado de satisfacción de los usuarios, podemos establecer el perfil en términos de calidad del servicio de EE. La localización en el territorio de los valores obtenidos, permite cotejar coincidencias entre las áreas que acusan baja calidad del mismo con aquellas áreas de crecimiento no planificado. La figura 13 muestra las áreas homogéneas con los diferentes niveles de calidad, estableciéndose zonas urbanas con requidades coincidentes con la zona de mayor crecimiento espontáneo.

b- Servicio de Gas Natural (GN).

La evaluación de este servicio se realizó bajo los mismos criterios explicitados en el servicio de EE. De igual manera calificamos esta fuente de energía a través de valorar sus cualidades, el factor de cobertura correspondiente al tendido de la red, y con el grado de aceptación de los usuarios. 


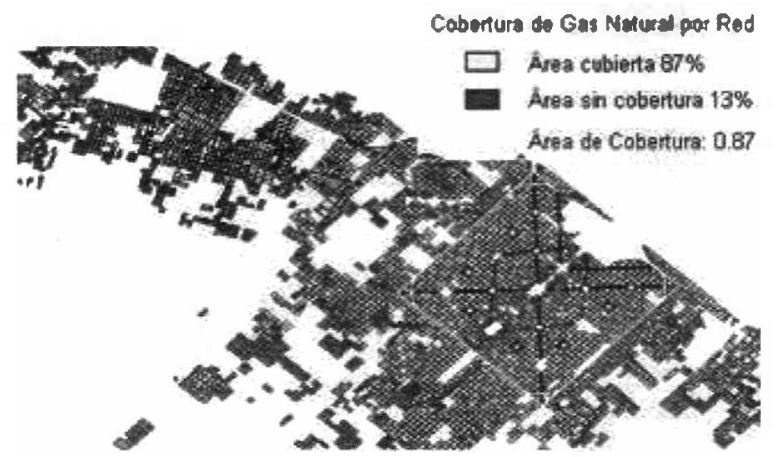

Figura 14: Cobertura de GN. $87 \%$ de cobertura. Adaptado de: G1. IIPAC

En cuanto a las cualidades del servicio de GN se consideran las mismas que en el servicio de EE, y su evaluación responde a los mismos criterios. Con respecto al área de cobertura, su red

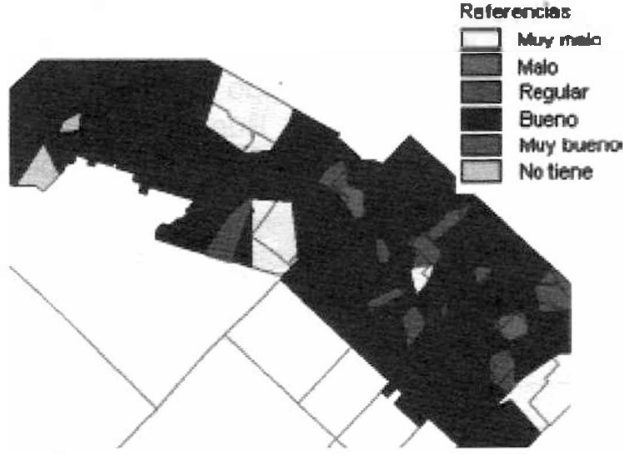

Figura 15: Áreas homogéneas de opinión. Red de GN. Adaptado de: G1. IIPAC

de distribución afecta actualmente al $87 \%$ de la población consolidada. (Figura 14).

El grado de aceptación del servicio de GN se evaluó a través de la opinión general de los usu-

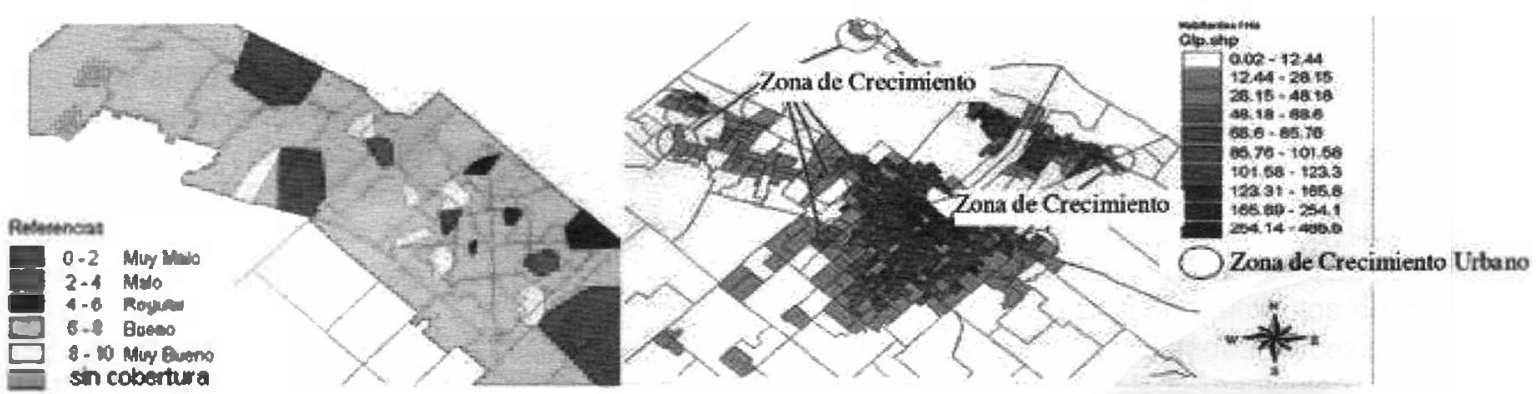

Figura 16: Pertil de Calidad del Servicio de GN. Inequidades coincidentes. Adaptado de: G1. IIPAC

arios, en donde se procesó estadísticamente la encuesta de hogares y se expandió territorialmente la muestra obteniendo un factor de opinión, normalizado representado en la figura 15.

Así como se realizó para el servicio de EE, se obtuvo el perfil de calidad del servicio de GN (Figura 16). Las áreas que registran mayores falencias coinciden al igual que en el servicio de EE, con las zonas de mayor crecimiento de población. Los resultados obtenidos marcan tendencias en cada una de las áreas valoradas las cuales pueden ser revertidas a partir del reconocimiento de las mismas.

El análisis de la demanda localizada de los usuarios y la correspondiente oferta de los servicios nos han permitido identificar áreas con problemas, y evaluar los desequilibrios generados por el crecimiento residencial espontáneo.

\section{Conclusiones}

Los resultados obtenidos muestran que el análisis integrado, abierto y flexible y la metodología propuesta es eficaz en cuanto a la evaluación de los sectores urbanos y sus servicios. La misma ha demostrado sensibilidad de resolución en cuanto a la obtención de información calificada y a la conformación de áreas homogéneas descriptivas de cada situación. Debemos remarcar que las respuestas visualizadas en los mapas marcan tendencias de equidad e inequidad urbana en cada una de las áreas valoradas, y sus límites responden a la precisión y localización de la información primaria.

Por otro lado, las herramientas previstas (índices, perfiles, mapas) nos permiten localizar las tendencias de comportamiento de cada servicio de infraestructura (EE y GN) en términos de calidad, verificar e identificar los diferentes aspectos a partir de la participación significativa de la demanda (usuarios) a través de la opinión y visualizar zonas de vulnerabilidad urbana. Se ha podido verificar coincidencias entre aquellos sectores que registran inequidades en su oferta, con los que registran crecimientos de población y demandas no previstas.

En cuanto a las consecuencias ambientales, el análisis permite establecer las áreas con mayor vulnerabilidad fundamentalmente en aquellos sectores con mayor consolidación urbana.

Para concluir, entendemos que el análisis propuesto y su instrumentación aportan información significativa para la planificación urbana. Las po- 
Sitilidades de identificar y verificar aéreas urbanas vulnerables en función de los crecimientos urvanos no previstos, conllevan a la necesidad de avanzar en el análisis de la causas (atractores) a los efectos poder prever las tendencias de crecimiento y ajustar la planificación de la oferta.

\section{Referencias}

A-selrad, H. (1999). Sustentabilidad y ciudad. FEvista Latinoamericana de Estudios Urbanos Ėgionales, Vol. 25, $N^{\circ} 74$. Print ISSN 02507161. EURE. Santiago.

$\therefore$ UDIBAIRES. Investigación realizada por Conz:so Nacional organizado por la CIC y Secre- E- a de Energía de la Nación. Contrato SE-N1- $\$ 99 / 83.1986$.

Conferencia Mundial Sobre el Medio Ambiente, sGenda XXI. (1992). Capítulo 9: Protección de la Atmósfera, Área: Desarrollo Sostenible. Río ¿ Janeiro, Brasil.

Desjeux D., Berthier C., Jarraffoux S., Orhant I. Taponier S. (1996). Anthropologie de I -électricit. Les objets électriques dans la vie quotidienne en France. L $\mathrm{HHarmattan}$. Logiques Sxciales.ISBN: 2-7384-4108-4. París.

Discoli C. (2003). Proyecto Sistema de diagnóstico de necesidades básicas en infraestuctura, servicios y calidad ambiental para centros urbanos o sectores con demandas insatisfechas. Financiado por la Agencia Nazonal de Promoción Científica y Tecnológica, ANPCYT. PICT 1314509. Argentina.

Discoli, C. Dicroce, L., Barbero, D., Amiel, J., San Juan, G., Rosenfeld, E. (2006a).Modelo de calidad de vida urbana. Formulación de un sistema de valoración de los servicios urbanos básicos de infraestructura aplicando lógica borrosa. Avances en energías renovables y meco ambiente, Vol. 10, pp. 21-28. CD, ISSN 03295184

Discoli, C., Rosenteld, E., San Juan, G., Martini, I.. Barbero, D., Ferreyro, C., Dicroce, L. (2006b). Urban Integration and Desintegration Forces: The habitants/users perception in a urban life quality model for the surroundings of La Plata. Buenos Aires, Argentine. 42 nd ISoCaRP Congress: Cities between Integration and Desintegration: Opportunities and Challenges, Eszmbul, 14-18 septiembre, 11 p., CD, ISBN 90$75524-45-5$.

C. Discoli, I. Martini, L. Dicroce, J. Ramirez Casas, J. Esparza, B. Brea, G. San Juan, E. Rosenfeld. (2007). Desarrollo metodológico para la dimensión de la opinión en el marco de un modelo de calidad de vida urbana. Avances en
Energías Renovables y Medio Ambiente, Vol. 11, pp. 0.1.97-01.104, CD, ISBN 0329-5184.

Díscoli, C., San Juan, C. Rosenfeld. E., Martini, I., Dicroce, L., Barbero, D., Ferreyro, C., Viegas, G., Ramírez Casas, J., Melchiori, M., Brea, B. (2008). Modelo de calidad de vida urbana. Desarrollo metodológico orientado a establecer tendencias de comportamiento entre la oferta de la ciudad y las demandas de sus usuarios. ENTAC (XII encontro nacional de tecnología do ambiente construido). Octubre 2008. Fortaleza. Brasil. CD. ISBN 978-85-89476-27-4.

Dupuy, G. (1991). L oUrbanisme des Réseaux. Théories et méthodes. Armand Colin, Paris.

Hardoy, J. (1993). Urbanización, sociedad y medio ambiente. En F. Goin y C. Goñi (eds.). Elementos de política ambiental. H. Cámara de Diputados de la Provincia de Buenos Aires. 983 p. ISBN978-99510-0-X. 70. 821-847. La Plata.

Observatorio de Calidad de Vida, La Plata (2001). Diagnóstico de calidad de vida en el partido de La Plata. Programa de Observatorio de Calidad de Vida. Secretaría de Extensión Universitaria. UNLP. La Plata.

Programa conjunto UNDP/Banco Mundial/UNCHS, Hábitat, 1991.

Rosenfeld, Y., Discoli, C., Martini, I., Hoses, S., Olivera, H., San Juan, G., Czajkowski, J., Rosenfeld, E. (2000). Formulación de instrumentos para la recolección y procesamiento de datos aplicado al estudio de redes edilicias y de infraestructura urbana. Poster, VIII Encontro Nacional de Tecnologia do Ambiente Construído, Salvador de Bahía, Brasil, 26 al 28 de abril de 2000, 8p. Artículo completo, Anais del VIII Encontro Nacional de Tecnología do Ambiente Construido, ENTAC 2000, (editado en CD-ROM).

Sureda, V. (2000). Sistema municipal de indicadores de sostenibilidad. P. 19. Barcelona, España.

URE-AM, Políticas de Uso racional de la Energia en Áreas Metropolitanas y sus efectos en la dimensión Ambiental, PID-CONICET N ${ }^{\circ}$ 4717/96. 1997/2000. 